\title{
Cálculo de elementos de vidrio en edificación: propuesta de norma española
}

\author{
A. FERNÁNDEZ CANTELI ${ }^{1}$, M.A. GARCÍA PRIETO' ${ }^{1}$, M.J. LAMELA REY ${ }^{1}$, E. CASTILLO RON² \\ 'EPS de Ingeniería de Gijón. Universidad de Oviedo. Campus de Viesques. 33203 Gijón \\ ${ }^{2}$ ETS de Ingenieros de Caminos, Canales y Puertos. Universidad de Cantabria. 39005 Santander
}

\begin{abstract}
El presente artículo describe un modelo probabilístico de cálculo de placas de vidrio y el procedimiento que permite la determinación de la carga admisible en determinados tipos de vidrio, como propuesta de una nueva norma española de vidrio estructural. Una vez caracterizado el material, el modelo, implementado en el programa Windesign, permite el cálculo de la probabilidad de fallo para una placa de vidrio monolítico de dimensiones dadas sometida a una determinada carga, o en su caso, la determinación del espesor de placa capaz de resistir una carga conocida para una probabilidad de fallo dada. Asimismo se presenta un estudio comparativo entre los resultados obtenidos para el modelo aquí propuesto y varias normas extranjeras de vidrio, tales como la norma americana ASTM E 1300-02, la italiana UNI 7143-72, la francesa NFP 78-201-1 y la neozelandesa NZS 4223.
\end{abstract}

Palabras clave: Vidrio estructural, Modelo probabilístico, Mecánica de fractura, Norma de cálculo.

Design of glass elements in building: A proposal for the Spanish code.

This paper reports on a probabilistic model for design of glass plates and the procedure to determine the allowable load of specified types of glass, as a proposal for a new Spanish code for structural glass. After the experimental material characterization, the design model, implemented in the Windesign software, allows the user to calculate either the probability of failure of a monolithic glass plate for a given dimensions and specific loading, or to determine the plate thickness needed to withstand a known loading for a given probability of failure. Moreover, a comparative analysis among the results obtained by the model proposed here and the current foreign glass design standards, such as the American ASTM E 1300-02, the Italian UNI 7143-72, the French NFP 78-201-1 and the New Zealander NZS 4223 is presented.

Keywords: Structural glass, Probabilistic model, Fracture mechanics, Design code.

\section{INTRODUCCIÓN}

En las últimas décadas el uso del vidrio en la construcción ha experimentado un notable aumento, pasando de desempeñar una función secundaria, como elemento de ventanas, a una función estructural primaria en vigas, soportes y muy especialmente en fachadas de edificios y de otras construcciones singulares e industriales. Aunque la incorporación del vidrio como material estructural permite conseguir efectos estéticos importantes en las estructuras modernas, su comportamiento frágil, debido a la inevitable presencia de microgrietas en su superficie y la posibilidad de una eventual rotura con resultados imprevisibles (1), exige aplicar métodos de cálculo rigurosos para alcanzar los niveles de seguridad exigidos en las normas de edificación habituales en otros materiales convencionales (2).

Por tanto, dado que la resistencia del vidrio, como material frágil, depende estrechamente de la presencia de defectos en su superficie, del tamaño del elemento y de la solicitación aplicada, el diseño y cálculo de elementos estructurales de vidrio deben estar basados indiscutiblemente en consideraciones probabilísticas y en criterios de la Mecánica de Fractura.
Recientemente el Ministerio de Fomento promovió un ambicioso programa para desarrollar el nuevo Código Técnico Español en la Edificación, relativo tanto al dimensionamiento de materiales convencionales como a nuevos materiales. En este marco de trabajo, se planteó la necesidad de formular una norma para el cálculo de elementos de vidrio y se ofreció al grupo de investigación de los autores de este artículo la oportunidad de colaborar con el Instituto de Ciencias de la Construcción "Eduardo Torroja" (CSIC) en la definición de las bases de cálculo y las aplicaciones estructurales del vidrio monolítico y laminado bajo condiciones de seguridad.

El cálculo de la resistencia de los materiales frágiles se puede plantear bajo dos perspectivas diferentes, correspondientes a un modelo basado en las tensiones críticas del material y a otro modelo basado en los tamaños máximos de defecto $(3,4)$. Aunque ambos modelos se fundamentan esencialmente en las mismas hipótesis, difieren en los parámetros de referencia considerados para establecer el criterio de rotura del material. Una vez caracterizado experimentalmente el material, ambos procedimientos 
permiten determinar la probabilidad de fallo de placas de vidrio para diferentes relaciones geométricas, solicitaciones de carga y condiciones de apoyo.

Un ejemplo del modelo basado en tensiones críticas es el desarrollado en el programa CARES de la NASA Glenn Research Center (5). En este modelo se describe el proceso de fallo del material fenomenológicamente, a través de la caracterización de la resistencia local del material y adoptando la tensión crítica como el parámetro de referencia para establecer la supervivencia del elemento considerado.

En el caso del modelo basado en el tamaño máximo de defectos (6), desarrollado por los autores en colaboración con la empresa Saint Gobain Cristalería, S.A. e implementado en el programa Windesign, se describe el mecanismo de fallo en base a consideraciones de la Mecánica de la Fractura. De esta forma, el modelo ofrece una interpretación física del criterio de fallo más detallada que el modelo de tensiones críticas, pero como contrapartida presenta un proceso más complejo de caracterización del material al precisar la determinación de la distribución de tamaños máximos de defecto.

En este artículo se presenta un procedimiento alternativo denominado modelo mixto (7), que aúna los modelos anteriores al deducir la función de distribución de tamaños máximos de defecto a partir de la distribución de tensiones críticas obtenidas experimentalmente. Asimismo el modelo mixto se aplica a dos ejemplos prácticos para la determinación de la carga admisible y el espesor necesario en placas rectangulares de vidrio monolítico para una probabilidad característica de fallo fijada en el $5 \%$.

\section{ANÁlISIS COMPARATIVO DE LA NORMATIVA VIGENTE}

Previamente al desarrollo de la propuesta de norma española sobre vidrio estructural, se realizó un estudio pormenorizado de algunas normas extranjeras actualmente vigentes, concretamente las normas americana ASTM E 1300-02 (8), italiana UNI 7143-72 (9), francesa NFP 78-201-1 (10) y neozelandesa NZS 4223 (11).

\subsection{Norma americana ASTM E 1300-02}

La norma ASTM E 1300-02 "Standard Practice for Determining Load Resistance of Glass in Buildings", que se encuentra en un continuo proceso de desarrollo, presenta un procedimiento de cálculo de resistencia de vidrios monolíticos, laminados y aislantes en placas rectangulares de espesor determinado, apoyadas en un único borde (placa en voladizo), en dos bordes (viga simplemente apoyada), en tres y cuatro bordes, sometidas a una carga lateral uniforme de corta o larga duración para una probabilidad de fallo dada del $8 \%$ o con la opción de cambiar a otros valores.

Esta norma, basada en el modelo de rotura de Beason (12), que relaciona la resistencia del vidrio con la condición de su superficie, presenta doce ábacos para la selección del espesor de placas de vidrio monolítico en un rango de $2,5 \mathrm{~mm}$ a $22 \mathrm{~mm}$.

De las normas consultadas, la ASTM es la más completa debido a la consideración de una gran variedad de tipos de vidrio, condiciones de apoyo y duración de la solicitación aplicada. Sin embargo, como puntos débiles es necesario mencionar que en esta norma no se especifican claramente los factores parciales de seguridad del material y de las cargas, no se tratan diferentes geometrías de placas, ni tipos de solicitación y no se establece un modelo probabilístico para el vidrio laminado.

\subsection{Norma italiana UNI 7143-72}

La norma UNI 7143-72“Vetri piani. Spessori dei vetri piani per vetrazioni in funzione delle loro dimensioni, dell'azione de vento e del carico neve", que permite la selección del espesor necesario en placas de vidrio de fachadas y cubiertas, está basada en la teoría elástica general de placas de Timoshenko (13), incluyendo un factor de corrección que tiene en cuenta el coeficiente de Poisson del material.

Para este cometido, se presentan cuatro ábacos para placas rectangulares de vidrio monolítico básico, apoyadas en sus cuatro bordes y expuestas a una carga lateral uniforme de viento o nieve. Mediante un factor de corrección basado en la relación de tensiones admisibles, la norma permite también seleccionar el espesor en placas de otros tipos de vidrio, diferentes al básico monolítico. En estos ábacos el rango de cargas considerado es de $490 \mathrm{~Pa}$ a $1230 \mathrm{~Pa}$.

Entre las ventajas de la norma italiana se encuentran la sencillez de uso y la posibilidad de cálculo de placas en posiciones distintas a la vertical.

Sin embargo, presenta como inconvenientes su antigüedad, pues se trata de una norma editada en 1972, y que no está basada en el cálculo de los estados límites. Asimismo se detectan importantes limitaciones en el uso de geometrías, tipos de cargas aplicadas y condiciones de apoyo de las placas, y no se especifica la probabilidad de fallo, dando resultados de dimensionamiento excesivamente conservadores.

\subsection{Norma francesa NFP 78-201-1}

La norma NFP 78-201-1 "Travaux de miroiterie vitrerie", publicada en 1993, está también basada en la teoría elástica general de placas de Timoshenko (13) y ofrece la posibilidad de seleccionar el espesor de placas planas de vidrio monolítico con dos, tres o cuatro bordes apoyados, expuestas a carga de viento o carga de nieve para el caso de placas de cubierta.

Entre sus principales ventajas se encuentran la simplicidad de uso, la posibilidad de calcular distintos tipos de vidrio, incluido el vidrio laminado y el vidrio aislante mediante factores tabulados, y la consideración de diferentes condiciones de apoyo.

Además, como ocurría con la norma italiana, tampoco existe en este caso una especificación clara de la probabilidad de fallo, ni el cálculo está basado en el principio de los estados límites.

\subsection{Norma neozelandesa NZS 4223}

The NZS 4223 "Glazing in Buildings. Part-4 Dead, Wind and Snow Loadings" se trata de una norma moderna que, en base a los estados límites de carga, permite realizar el cálculo del espesor mínimo necesario de placas de distinto tipo de vidrio, tanto en posición vertical como inclinada, y con condiciones de apoyo a lo largo de dos o cuatro de sus bordes. Para la realización de este cálculo, la norma presenta doce ábacos combinando seis tipos distintos de vidrio con las dos condiciones de apoyo antes mencionadas.

Como aciertos más notables de esta norma se pueden apuntar la amplia selección de tipos de vidrio disponibles, la posibilidad de dimensionar placas verticales e inclinadas y la 
aplicación de la teoría de los estados límite. Sin embargo, sus resultados son conservadores debido a las simplificaciones adoptadas $\mathrm{y}$, por otro lado, en su procedimiento de cálculo no aparecen especificados ni la probabilidad de fallo, ni los factores de seguridad considerados.

\section{DESCRIPCIÓN DEL MODELO DE CÁLCULO}

En un primer momento, el modelo surgió con objeto de calcular elementos rectangulares de vidrio monolítico, simplemente apoyados en su contorno y sometidos a una carga lateral uniforme, equivalente a la solicitación de viento. La Figura 1 representa un esquema del modelo propuesto, con el que se trata de mantener un paralelismo con el dimensionamiento de materiales convencionales, como el acero y el hormigón, por lo que se consideran tres ámbitos de actuación denominados 'Resistencia', 'Solicitación' y 'Probabilidad de fallo'.

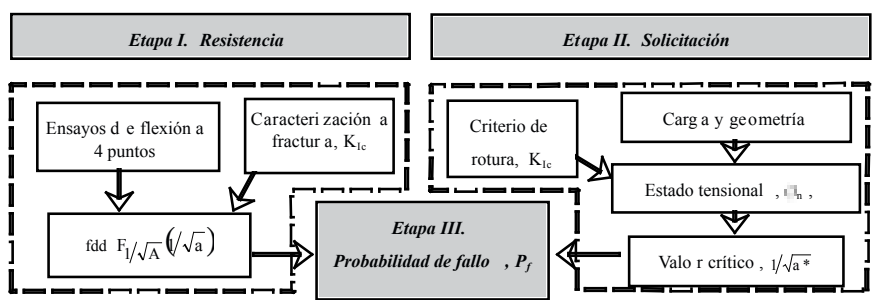

Fig. 1- Esquema del modelo propuesto de dimensionamiento de vidrio.

\subsection{Etapa I. Resistencia}

En esta primera etapa de cálculo, la caracterización del material se realiza mediante experimentación. Para conocer la resistencia del material se recurre a la realización de ensayos de flexión en cuatro puntos de carga, según el esquema de la Figura 2.

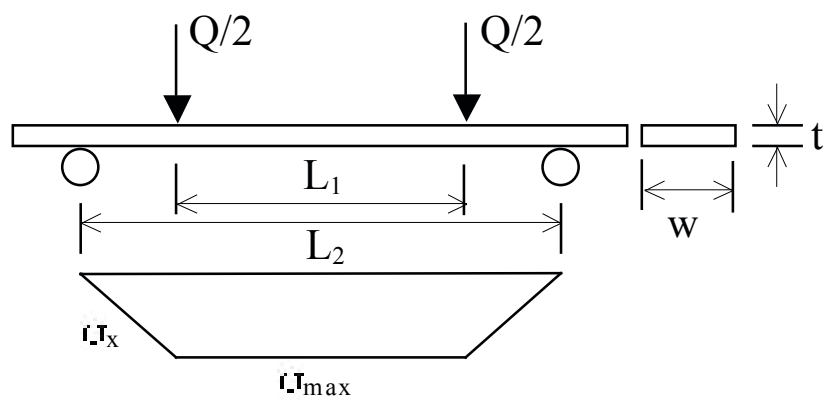

Fig. 2- Ensayo de flexión a cuatro puntos de carga.

De los resultados obtenidos y su evaluación estadística se obtiene la función de distribución de tensiones críticas en el material, $\sigma$, que se supone que sigue una ley de Weibull para valores extremos mínimos:

$$
\mathrm{F}_{\Sigma}(\sigma)=1-\exp \left[-\left(\frac{\sigma-\lambda_{\sigma}}{\delta_{\sigma}}\right)^{\beta_{\sigma}}\right] ; \quad \sigma \geq \lambda_{\sigma}
$$

siendo $\lambda_{\sigma} \delta_{\sigma}$ y $\beta_{\sigma}$ los parámetros de localización, escala y forma, respectivamente.
A partir de $\mathrm{F}_{\Sigma}(\sigma)$ y de la expresión general de la Mecánica de la Fractura [2], que relaciona la tensión, $\sigma$, con el tamaño de defecto, a, a través del factor de intensidad de tensiones, $\mathrm{K}$, y el factor geométrico adimensional, $\mathrm{f}_{1^{\prime}}$ correspondiente al tipo de defecto considerado (14)

$$
\mathrm{K}=\sigma \mathrm{f}_{1} \sqrt{\pi \mathrm{a}},
$$

se obtiene la expresión de una nueva función de distribución dependiente de la variable $1 / \sqrt{a}$, en la que $\mathrm{k}_{1}=\mathrm{K} / \mathrm{f}_{1} \sqrt{\pi}$ :

$$
\mathrm{F}_{\Sigma}(\sigma)=\mathrm{F}_{\Sigma}\left(\frac{\mathrm{k}_{1}}{\sqrt{\mathrm{a}}}\right)=1-\exp \left[-\left(\frac{\frac{\mathrm{k}_{1}}{\sqrt{\mathrm{a}}-\lambda_{\sigma}}}{\delta_{\sigma}}\right)^{\beta_{\sigma}}\right]
$$

Designando los nuevos parámetros por

$$
\lambda_{1 / \sqrt{\mathrm{a}}}=\frac{\lambda_{\sigma}}{\mathrm{k}_{1}}, \delta_{1 / \sqrt{\mathrm{a}}}=\frac{\delta_{\sigma}}{\mathrm{k}_{1}} \text { y } \beta_{1 / \sqrt{\mathrm{a}}}=\beta_{\sigma},
$$

la función de distribución obtenida, $F_{1 / \sqrt{A}}(1 / \sqrt{a})$, sigue también una ley de Weibull para valores mínimos con la siguiente expresión:

$$
\mathrm{F}_{1 / \sqrt{\mathrm{A}}}(1 / \sqrt{\mathrm{a}})=1-\exp \left[-\left(\frac{\frac{1}{\sqrt{\mathrm{a}}}-\lambda_{1 / \sqrt{\mathrm{a}}}}{\delta_{1 / \sqrt{\mathrm{a}}}}\right)^{\beta_{1 / \sqrt{\mathrm{a}}}}\right]
$$

Dada la gran dispersión de resultados de resistencia que caracteriza a los materiales frágiles, es obligada la consideración del efecto de escala en los cálculos. Para ello, se define como área de referencia, $\mathrm{A}_{\mathrm{ref}}$, el área equivalente de la superficie de la probeta que, sometida a tracción uniforme bajo la tensión máxima, $\sigma_{\text {max }}$ del ensayo de flexión a cuatro puntos, proporciona una función de distribución igual a la obtenida de la experimentación. Para el cálculo del área de referencia se ha deducido la siguiente expresión:

$$
\mathrm{A}_{\text {ref }}=\mathrm{wL}_{1}+\frac{\mathrm{w}\left(\mathrm{L}_{2}-\mathrm{L}_{1}\right)}{\left(\beta_{\sigma}+1\right)}\left[1-\frac{2 \mathrm{w} \mathrm{t}^{2} \lambda_{\sigma}}{3 \mathrm{Q}\left(\mathrm{L}_{2}-\mathrm{L}_{1}\right)}\right][6]
$$

en la que $w$ y $t$ representan el ancho y el espesor de la probeta, $\mathrm{L}_{1}$ y $\mathrm{L}_{2}$ las distancias entre la aplicación de la carga y los apoyos (ver Figura 2), $\lambda_{\sigma}$ y $\beta_{\sigma}$ los parámetros de la función de Weibull de tensiones críticas y $Q$ el valor de la carga de rotura.

\subsection{Etapa II. Solicitación}

A partir del estado tensional de la placa de vidrio sometida a carga lateral uniforme y de la adopción de un criterio de rotura apropiado, que exprese la relación entre las tensiones actuantes en el frente de grieta y su inestabilidad, se puede calcular el valor crítico de la variable considerada, 
$1 / \sqrt{\mathrm{a}} *$, en un punto y para una orientación determinada.

Mientras que el modelo permite que el estado tensional de la placa se calcule mediante el método de los elementos finitos, a través de un programa comercial, la elección del criterio de rotura adoptado, en principio, queda abierta. Entre los criterios de rotura propuestos en la bibliografía especializada, se optó aquí por el criterio de Shetty, cuya expresión es la siguiente:

$$
\frac{\mathrm{K}_{\mathrm{I}}}{\mathrm{K}_{\mathrm{IC}}}+\left(0,8 \frac{\mathrm{K}_{\mathrm{II}}}{\mathrm{K}_{\mathrm{IC}}}\right)^{2}=1 \text {, }
$$

siendo $\mathrm{K}_{\mathrm{I}}$ y $\mathrm{K}_{\mathrm{II}}$ los factores de intensidad de tensiones en modo I y modo II y $\mathrm{K}_{\mathrm{IC}}$ la tenacidad a fractura del material.

\subsection{Etapa III. Probabilidad de fallo}

Una vez definidos los estados de resistencia y solicitación, en esta última etapa del modelo se comparan ambos entre sí para evaluar la situación de seguridad o no-seguridad según un criterio probabilístico.

La probabilidad de fallo de un determinado elemento finito de la placa de vidrio se calcula como la probabilidad de tener en el material un valor de $1 / \sqrt{\mathrm{a}}$ menor o igual que el valor crítico $1 / \sqrt{\mathrm{a}^{*}}$. Al generalizar el cálculo de esta probabilidad a todos los elementos considerados en la placa, la probabilidad de rotura de dicha placa adopta la expresión siguiente, en la que se introduce la posibilidad de que la grieta esté orientada según varias direcciones:

$$
\mathrm{P}_{\mathrm{f}}(\text { placa })=1-\prod_{\mathrm{i}=1}^{\mathrm{n}} \mathrm{P}_{\mathrm{f}_{\mathrm{i}}}
$$

siendo $\mathrm{P}_{\mathrm{f}_{\mathrm{i}}}$ igual a:

$$
\left.\sum_{\mathrm{k}=1}^{\mathrm{p}} \frac{1}{\mathrm{p}}\left[1-\exp \left(-\frac{\mathrm{A}_{\mathrm{i}}}{\mathrm{A}_{\text {ref }}}\left(\frac{\frac{1}{\sqrt{\mathrm{a}_{\mathrm{i}}}-\lambda_{1} / \sqrt{\mathrm{a}}_{\mathrm{k}}}}{\delta_{1 / \sqrt{\mathrm{a}}_{\mathrm{k}}}}\right)\right)^{\beta 1 / \sqrt{\mathrm{a}}_{\mathrm{k}}}\right)\right] \text {, }
$$

donde: $\mathrm{n}$ y $\mathrm{p}$ representan el número de elementos y orientaciones de grieta considerados, $\lambda_{1 / \sqrt{a}_{\mathrm{k}}}, \delta_{1 / \sqrt{\mathrm{a}}_{\mathrm{k}}}$ y $\beta_{1 / \sqrt{\mathrm{a}}_{\mathrm{k}}} \quad, \mathrm{y}$ los parámetros de la función de Weibull para una orientación ' $\mathrm{k}$ ' de la grieta, $A_{i}$ el área del elemento ' $\mathrm{i}^{\prime}$ y $\mathrm{A}_{\text {ref }}$ el área de referencia calculada con la expresión [6].

\section{EJEMPLOS DE APLICACIÓN}

En este apartado se presentan dos ejemplos de dimensionamiento deducidos de los ábacos elaborados con ayuda del programa Windesign, de acuerdo con el procedimiento del modelo de cálculo expuesto anteriormente.

Ejemplo1. Se trata de determinar la carga lateral uniforme admisible de cuatro placas de vidrio monolítico recocido de $5 \mathrm{~mm}$ de espesor, simplemente apoyadas en el contorno y cuyas dimensiones se indican en la Figura 3 y en la Tabla I.

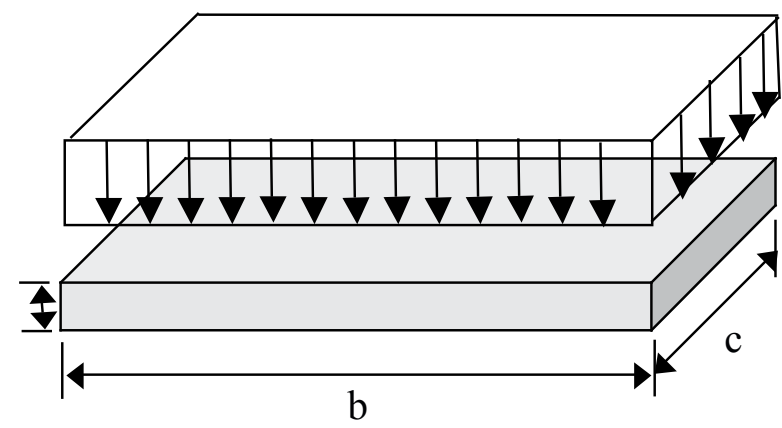

Fig. 3- Placa rectangular de vidrio sometida a carga lateral uniforme.

Para los cálculos se considerará una probabilidad de fallo del $5 \%$ y las siguientes características mecánicas del material: Módulo de Young $\mathrm{E}=7,3.10^{4} \mathrm{MPa}$, coeficiente de Poisson $v=$ 0,22 y tenacidad a fractura $\mathrm{K}_{\mathrm{IC}}=0,712 \mathrm{MPa} \cdot \mathrm{m}^{1 / 2}$.

En primer lugar, se estimó la función de distribución de Weibull de las tensiones críticas resultantes del programa experimental, , resultando unos parámetros: $\lambda_{\sigma}=36,69 \mathrm{MPa}$, $\delta_{\sigma}=116,66$ MPa y $\beta_{\sigma}=4,27$.

A continuación, a partir de $\mathrm{F}_{\Sigma}(\sigma)$ se dedujo la función dependiente del tamaño de defecto, a, denominada $\mathrm{F}_{1 / \sqrt{\mathrm{A}}}(1 / \sqrt{\mathrm{a}})$, con parámetros: $\lambda_{1 / \sqrt{\mathrm{a}}}=65,17 \mathrm{~m}^{-1 / 2}$, $\delta_{1 / \sqrt{\mathrm{a}}}=207,21 \mathrm{~m}^{-1 / 2}$ y, $\beta_{1 / \sqrt{\mathrm{a}}}=4,27$

calculados mediante las expresiones [4] y teniendo en cuenta que la constante $\mathrm{k}_{1}$ toma un valor de $0,563 \mathrm{MPa}$. $\mathrm{m}^{1 / 2}$ para el tipo de vidrio utilizado y $\mathrm{f}_{1}=0,713$, factor correspondiente a una grieta superficial semicircular (12).

TABLA I. CARGAS ADMISIBLES PARA LAS PLACAS DE VIDRIO EN ESTUDIO DE 5 MM DE ESPESOR.

\begin{tabular}{|c|c|c|c|}
\hline & $\mathrm{b}[\mathrm{m}]$ & $\mathrm{c}[\mathrm{m}]$ & $\mathrm{L}[\mathrm{Pa}]$ \\
\hline Placa 1 & 1,00 & 2,00 & 2450,95 \\
\hline Placa 2 & 1,40 & 2,00 & 1615,40 \\
\hline Placa 3 & 2,00 & 2,00 & 1157,63 \\
\hline Placa 4 & 3,00 & 2,00 & 718,09 \\
\hline
\end{tabular}

Con estos datos y el programa Windesign se elaboró el ábaco representado en la Figura 4, del que se obtuvieron las cargas admisibles para cada una de las placas en estudio, representadas en la Tabla I.

Ejemplo 2. Se trata de calcular el espesor mínimo de una placa de vidrio de dimensiones $1,8 \mathrm{~m} \times 2 \mathrm{~m}$ sometida a una carga lateral uniforme de $5 \mathrm{kPa}$. Se adoptará el mismo tipo vidrio del ejemplo anterior, así como una probabilidad de fallo del $5 \%$.

Tal como se muestra en la Figura 4, el espesor mínimo de placa, capaz de resistir la carga aplicada, ha de ser de 10 $\mathrm{mm}$.

Con el fin de completar el análisis comparativo de la normativa vigente expuesto en el segundo apartado de este artículo, en la Figura 5 se representan los valores de las cargas laterales admisibles obtenidas con la propuesta de norma 


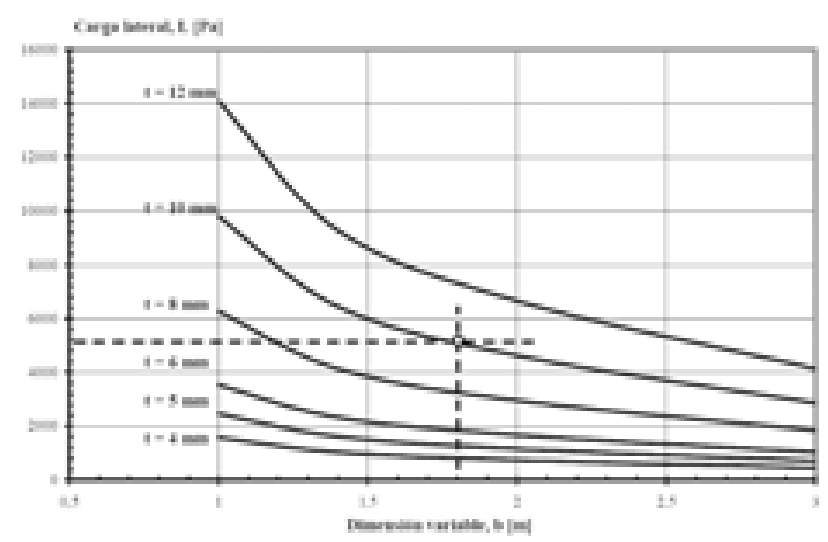

Fig. 4- Ábaco de cargas admisibles para una placa rectangular de dimensión fija c $=2 \mathrm{~m} \mathrm{y}_{\mathrm{f}}=5 \%$.

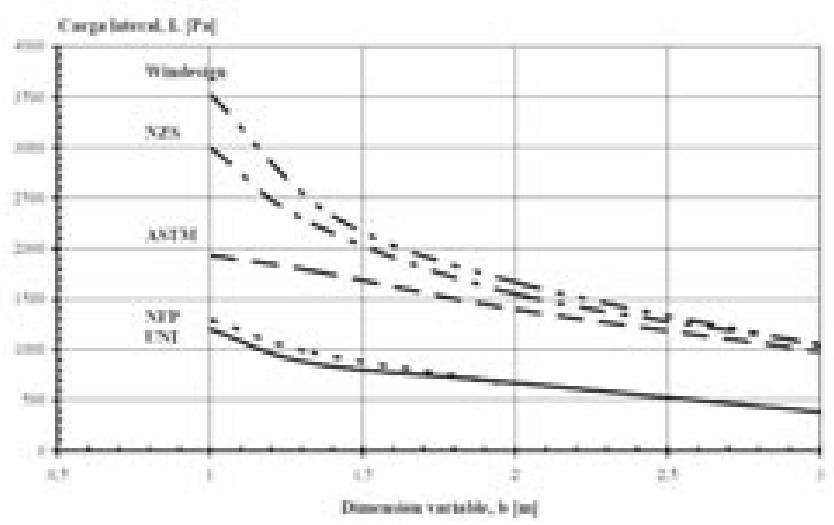

Fig. 5- Aplicación de distintas normas a una placa rectangular de 6 $\mathrm{mm}$ de espesor y dimensión fija $\mathrm{c}=2 \mathrm{~m}$.

de los autores (Windesign) y las normas internacionales analizadas para una placa de vidrio rectangular de $6 \mathrm{~mm}$ de espesor y dimension fija $\mathrm{c}=2 \mathrm{~m}$.

En esta gráfica se observa una tendencia común en todas las normas consideradas, si bien con la aplicación de distintos coeficientes de seguridad, supuestamente debido al diferente concepto de probabilidad de fallo al que están referidas.

\section{CONCLUSIONES}

De acuerdo con lo expresado anteriormente, las conclusiones de este trabajo son las siguientes:

1.- El modelo de cálculo de vidrio propuesto supone una alternativa al modelo tradicional basado en tensiones críticas y proporciona una visión más intuitiva de la fractura del material.

2.- Ambos modelos pueden considerarse como metodologías equivalentes para el cálculo de vidrio, dado que están basados en hipótesis similares.

3.- La comparación de las normas de vidrio estudiadas permite deducir que el modelo de dimensionamiento propuesto interpreta adecuadamente el comportamiento a rotura de placas de vidrio, aunque se hace necesario aplicar unos coeficientes de seguridad apropiados.
4.- En los ábacos propuestos para una futura norma española, relativa al cálculo de vidrio estructural, se hace imprescindible la definición de unos coeficientes de seguridad, que deberán ser estimados empíricamente o deducidos por comparación con otras normas vigentes a nivel internacional.

5.- La comparación cuantitativa con otras normas extranjeras resulta compleja ya que, en general, no especifican los valores de los coeficientes de seguridad empleados y, por otro lado, algunas no se basan en modelos probabilistas, ni tienen en cuenta la teoría no-lineal de placas.

6.- El modelo de cálculo propuesto en este artículo para vidrio monolítico puede hacerse extensivo al cálculo de vidrio laminado y vidrio aislante.

\section{AGRADECIMIENTOS}

Los autores agradecen el apoyo económico recibido a través del proyecto y contrato de investigación CC-99-MAT1017-C0302 y CN-03-088, subvencionados respectivamente por el Ministerio de Ciencia y Tecnología y el Ministerio de Fomento.

\section{BIBLIOGRAFÍA}

1.W. Suchanek, M.Yoshimura,"Processing and Properties of HydroxyapatiteJ.D. Wörner, J. Schneider, A. Fink. «Glasbau, Grundlagen, Berechnung, Konstruktion». Springer (Alemania) 2001.

2. R. Claramunt, S. Postigo, R. Perera, A. Pacios, A. Ros, C. Huerta. «Seguridad ante impacto humano de acristalamientos en edificios». Bol. Soc. Esp. Cerám. V., 44 [5] 286-290 (2005).

3. M.A. García Prieto. "Resumen de tesis doctoral: Dimensionamiento probabilístico y análisis experimental de vidrios en rotura». Bol. Soc. Esp. Cerám. V., 40 [5] 389 (2001).

4. J. Pascual Cosp, A.J. Ramírez del Valle, J. Zapatero Arenzana, J.C. Galiano Serrano. "Análisis estadístico de la resistencia mecánica de materiales cerámicos de mullita sometidos a diferentes ciclos de calentamiento». Bol. Soc. Esp. Cerám. V., 44 [6] 415-420 (2005).

5. N.N. Nemeth, J.M. Manderscheid, J.P. Gyekenyesi. «Ceramics Analysis and Reliability Evaluation of Structures (CARES)». NASA Tech. Paper 2916 (EEUU) 1990.

6. M.A. García-Prieto. «Dimensionamiento probabilístico y análisis experimental de vidrios en rotura». Tesis Doctoral. Universidad de Oviedo (España) 2001.

7. A. Fernández-Canteli, M.A. García-Prieto, M.J. Lamela, E. Real. «Design of Structural Glass Elements Using a Probabilistic Model». Int. Symposium on Developments in Plasticity and Fracture. Centenary of Huber Criterion (Poland) 2004.

8. ASTM E 1300-02 «Standard Practice for Determining Load Resistance of Glass in Buildings» (EEUU) 2002.

9. UNI 7143-72 «Vetri piani. Spessori dei vetri piani per vetrazioni in funzione delle loro dimensioni, dell'azione de vento e del carico neve» (Italia) 1972.

10. NFP 78-201-1 «Travaux de miroiterie vitrerie» (Francia) 1993.

11. NZS 4223: 2000 «Glazing in Buildings. Part-4 Dead, Wind and Snow Loadings» (Nueva Zelanda) 2000.

12. W.L. Beason, T.L. Kohutek, J.M. Bracci. «Basis for ASTM E-1300 Glass Thickness Selection Procedure». Texas A\&M University (EEUU) 1996.

13. S. Timoshenko, S. Woinowsky-Krieger. "Teoría de placas y láminas». Ediciones Urmo (España) 1959.

14. H.L. Ewalds, R.J.H. Wanhill. «Fracture Mechanics». Edward Arnold Ed. (The Netherlands) 1993.

Recibido: 11.02 .05

Aceptado: 22.11 .05 